\title{
23. Boundary Value Problem on Symmetric Homogeneous Spaces
}

\author{
By Toshio Oshima*) and Jiro SekIGUCHI**)
}

(Communicated by Kôsaku YosidA, M. J. A., June 14, 1977)

1. Introduction. Any eigenfunction of all invariant differential operators on a Riemannian symmetric space can be represented by the Poisson integral of a hyperfunction on its Martin boundary (cf. [2], [3]). We can also formulate a boundary value problem for a little wider class of (not necessarily Riemannian) symmetric spaces. For examples : $S L(n, \boldsymbol{R}) / S O(p, n-p), S p(n, \boldsymbol{R}) / U(p, n-p), S p(n, \boldsymbol{R}) / G L(n, \boldsymbol{R})$. Our theorem in this paper is a natural generalization of the result in [2] and [3] under a certain mild condition.

2. Notation. Let $G$ be a connected real semisimple Lie group with finite center, $K$ a maximal compact subgroup of $G$. Let $g$ be the Lie algebra of $G, \mathfrak{t}$ the Lie subalgebra of $K$ in $g$. Let $\theta$ be the compatible Cartan involution of $g$. Let $\mathfrak{a}$ be a maximal abelian subspace in $\mathfrak{p}=\{X \in \mathfrak{g} ; \theta(X)=-X\}, \mathfrak{a}^{*}$ its dual and $\mathfrak{a}_{c}^{*}$ the complexification of $\mathfrak{a}^{*}$. Let $\Sigma$ be the restricted root system of $(\mathfrak{g}, \mathfrak{a})$ and let us introduce an order in $\Sigma$. We denote by $\Psi=\left\{\alpha_{1}, \cdots, \alpha_{l}\right\}$ the set of positive simple roots in this order. Put $\mathfrak{g}^{\alpha}=\{X \in \mathfrak{g} ;[H, X]=\alpha(H) X$ for any $H$ in $\mathfrak{a}\}$ and let us denote by $\rho$ one-half of the sum of positive roots. Furthermore, let $G=K A N$ be the compatible Iwasawa decomposition, $M$ the centralizer of $\mathfrak{a}$ in $K, M^{*}$ the normalizer of $\mathfrak{a}$ in $K$, and $\mathfrak{m}$ and $\mathfrak{n}$ the Lie algebras of $M$ and $N$, respectively. The quotient group $W=M^{*} / M$ is called the Weyl group.

3. Preliminary results. We will define the symmetric space $G / K_{s}$ where we will investigate simultaneous eigenfunctions of the invariant differential operators.

Definition 1. We call the mapping $\varepsilon: \Sigma \rightarrow\{-1,1\}$ a signature of roots if the followings are satisfied.

(i) $\varepsilon\left(\alpha_{i}\right) \in\{-1,1\}$ for $\alpha_{i} \in \Psi$.

(ii) $\varepsilon(\alpha)=\varepsilon\left(\alpha_{1}\right)^{m_{1}} \cdots \varepsilon\left(\alpha_{l}\right)^{m_{l}}$ for $\alpha=\sum_{i=1}^{l} m_{i} \alpha_{i} \in \Sigma$.

For a given signature $\varepsilon$, we can associate an involutive automorphism $\theta_{s}$ of $\mathrm{g}$ by the following :

Definition 2. We define the involutive automorphism $\theta_{s}$ of $\mathrm{g}$ so that the conditions

\footnotetext{
*) Department of Mathematics, University of Tokyo.
}

**) Department of Mathematics, Kyoto University. 
(i) $\theta_{s}(X)=\varepsilon(\alpha) \theta(X)$ for $X \in \mathfrak{g}^{\alpha}(\alpha \in \Sigma)$,

(ii) $\theta_{s}(X)=\theta(X)$ for $X \in \mathfrak{m}+\mathfrak{a}$

hold and call it the $\varepsilon$-involution of $\mathrm{g}$.

Put $\mathfrak{f}_{\mathrm{s}}=\left\{X \in \mathrm{g} ; \theta_{\mathrm{s}}(X)=X\right\}$, let $K_{\mathrm{s}}^{0}$ be the analytic subgroup of $G$ generated by $\mathfrak{f}_{\mathrm{s}}$ and put $K_{\mathrm{s}}=M K_{\mathrm{s}}^{0}$. Furthermore, put $M_{\mathrm{s}}^{*}=M^{*} \cap K_{\mathrm{s}}$ and $W_{s}=M_{*}^{*} / M$ and let $w_{1}=e, w_{2}, \cdots, w_{r}$ be representatives of the quotient $W_{s} \backslash W$, where $r=\left[W: W_{\imath}\right]$. We will fix $\bar{w} \in M^{*}$ so that $w=\bar{w} M$ for any $w \in W$.

Lemma 3. 1) $\mathfrak{g}=\mathfrak{f}_{\mathrm{s}}+\mathfrak{a}+\mathfrak{n}$ (direct sum).

2) $G \supset \bigcup_{i=1}^{r} K_{\varepsilon} \bar{w}_{i} A N$ (open dense, unique, disjoint).

Remark. 1) The complexifications of $\mathfrak{f}$ and $\mathfrak{f}_{\mathrm{s}}$ are isomorphic in the complexification of $\mathrm{g}$.

2) If $\varepsilon$ is the trivial signature, then $K_{s}$ equals $K$ and $G / K_{c}$ is a Riemannian symmetric space. But $G / K_{c}$ is not necessarily Riemannian in general.

4. Poisson transform. Let $D\left(G / K_{s}\right)$ (resp. $D(G / K)$ ) be the ring of invariant differential operators on $G / K_{s}$ (resp. $\left.G / K\right)$.

Lemma 4. $D\left(G / K_{s}\right) \cong D(G / K)$.

For a real analytic manifold $X$, we denote by $\mathscr{B}(X)$ the space of hyperfunctions on $X$ (cf. [4]). Put

$\mathscr{B}\left(G / K_{s} ; \mathscr{H}(\chi)\right)=\left\{u \in \mathscr{B}\left(G / K_{s}\right) ; D u=\chi(D) u\right.$ for any $\left.D \in D\left(G / K_{s}\right)\right\}$ for any algebra homomorphism $\chi$ of $D\left(G / K_{\mathrm{s}}\right)$ into $C$ and put

$$
e^{\lambda\left(H_{s}^{i}(g)\right)}= \begin{cases}e^{\lambda(\log a)} & \text { if } g=k_{\mathrm{s}} \bar{w}_{i} a n, k_{\mathrm{s}} \in K_{\mathrm{s}}, a \in A, n \in N, \\ 0 & \text { if } g \notin K_{\mathrm{s}} w_{i} A N\end{cases}
$$

for any $\lambda \in \mathfrak{a}_{c}^{*}$ and any $g \in G(i=1, \cdots, r)$.

Lemma 5. The function $e^{\lambda\left(H_{s}^{i}(g)\right)}$ on $G$ is a hyperfunction with the meromorphic parameter $\lambda \in \mathfrak{a}_{c}^{*}$. Its poles are contained in the set $\left\{\lambda \in \mathfrak{a}_{c}^{*} ; \frac{2\langle\lambda, \alpha\rangle}{\langle\alpha, \alpha\rangle} \in Z\right.$ for some $\left.\alpha \in \Sigma\right\}$. If $\lambda$ does not belong to the set, we can take a unique algebra homomorphism $\chi_{\lambda}$ of $D\left(G / K_{s}\right)$ into $C$ such that $e^{-(\lambda+\rho) H_{s}^{i}(g-1)}$ is in $\mathcal{B}\left(G / K_{s} ; \mathscr{M}\left(\chi_{2}\right)\right)$ for $i=1, \cdots, r$. Here $\langle\cdot, \cdot\rangle$ denotes the inner product induced by the Killing form.

Next we define the space of hyperfunction sections on a line bundle over $G / P(P=M A N)$ for any $\lambda \in \mathfrak{a}_{c}^{*}$ :

$$
\mathscr{B}(G / P ; \lambda)=\left\{f(g) \in \mathscr{B}(G) ; f(\text { gman })=e^{(\lambda-\rho) \log a} f(g)\right.
$$

for $g \in G, m \in M, a \in A, n \in N\}$.

Then a partial Poisson transform of every $f(g)$ in $\mathcal{B}(G / P ; \lambda)$ is defined by

$$
\left(\mathcal{Q}_{\lambda, s}^{i} f\right)(g)=\int_{K} f(k) e^{-(\lambda+\rho) H_{s}^{i}(g-1 k)} d k
$$

with the normalized Haar measure $d k$ on $K(i=1, \cdots, r)$. We can prove that the partial Poisson transform $\mathcal{Q}_{\lambda, s}^{i}$ is a $G$-homomorphism of $\mathscr{B}(G / P ; \lambda)$ into $\mathscr{B}\left(G / K_{s} ; \mathscr{M}\left(\chi_{\lambda}\right)\right)$. 
Now we can state our theorem.

Theorem. Assume $a \lambda$ in $\mathfrak{a}_{c}^{*}$ satisfies the condition

$$
\frac{2\langle\lambda, \alpha\rangle}{\langle\alpha, \alpha\rangle} \notin Z \quad \text { for any } \alpha \text { in } \Sigma \text {. }
$$

Then the Poisson transform

$$
\mathcal{Q}_{\lambda, s}: \stackrel{r}{\oplus} \mathcal{B}(G / P ; \lambda) \rightarrow \mathscr{B}\left(G / K_{s} ; \mathscr{M}\left(\chi_{\lambda}\right)\right)
$$

defined by $\mathcal{Q}_{\lambda, \varepsilon}\left(f_{1}, \cdots, f_{r}\right)=\sum_{i=1}^{r} \mathcal{Q}_{\lambda, s}^{i} f_{i}$ is an onto G-isomorphism.

We will describe the idea of the proof of the theorem. First we embed the symmetric space $G / K_{s}$ with its Martin boundaries in a compact real analytic manifold. We remark that there are $r=\left[W: W_{s}\right]$ Martin boundaries in the closure of $G / K_{s}$ in this realization. Then it is an essential fact that the system of differential equations on $G / K_{\text {s }}$

$$
\mathscr{H}(\chi): D u=\chi(D) u \quad \text { for any } D \in D\left(G / K_{\varepsilon}\right)
$$

has regular singularity along the walls around every Martin boundary (cf. [1]). Using this fact, we can take the boundary values of any eigenhyperfunction of the invariant differential operators on the symmetric space $G / K_{c}$ to its $r$ Martin boundaries. We can prove that the two mappings, the Poisson transform and taking the boundary values of the eigenhyperfunctions, are mutually inverse mappings. Hence we can obtain the result.

This method of the proof is the same as in [2]. The precise argument will be published elsewhere.

\section{References}

[1] M. Kashiwara and T. Oshima: Systems of differential equations with regular singularities and their boundary value problems (to appear in Ann. of Math.).

[2] M. Kashiwara, A. Kowata, K. Minemura, K. Okamoto, T. Oshima, and M. Tanaka: Eigenfunctions of invariant differential operators on a symmetric space (to appear).

[ 3 ] K. Minemura and T. Oshima: Boundary value problems with regular singularity and Helgason-Okamoto conjecture. Publ. Res. Inst. Math. Sci., 12, Suppl., 257-265 (1977).

[4] M. Sato: Theory of hyperfunctions. I, II. J. Fac. Sci. Univ. Tokyo, Sect. I, 8, 139-193, 357-437 (1959). 